\title{
Intelligent Management of Traffic Crossroads in VANETs Environment Using Multi-Agent Systems
}

\author{
Said Benkirane ${ }^{1}$ and Ahmed Jadir ${ }^{2}$ \\ SAEDD Laboratory, High School of Technology, \\ Cadi Ayyad University, Essaouira, Morocco \\ 1sabenkirane@gmail.com \\ 2ah.jadir@gmail.com
}

\begin{abstract}
In recent years, Vehicular Ad hoc Networks (VANETs) have become one of the most relevant wireless technologies. They constitute one of the most promising approaches for the implementation of intelligent transportation systems (ITS) which is involved in several fields of activities: in optimizing the use of transport infrastructure, improving safety (including road safety) and enabling users to be better informed and safer as well as developing services. In this paper, we present an approach based on a Multi-Agent Systems in the VANET environment allowing a smart management of a crossroads. It makes the road traffic, in this sensitive place, more flexible and more efficient. Moreover, this approach helps to avoid afterwards enormous problems, such as, waste of energy and time as well as better the traffic jam in the congestion conditions.
\end{abstract}

Keywords — ITS, VANET, Multi-Agent Systems, Traffic Management, Crossroads.

\section{INTRODUCTION}

A VANET network is a particularity of MANET networks where mobile nodes are smart vehicles equipped with computers, network cards and sensors. Like any other Ad hoc network, vehicles can communicate with each other (for example: exchanging traffic information) or with base stations called roadside unit (RSU) which can be placed all along the roads (seeking information or accessing other networks ...) [1].

VANET networks are based on communication and information exchange between vehicles, and between vehicles and roadside unit (Examples: signals, intersections lights, etc.) or external network elements (Satellites, WiMAX, LTE...) (Fg.1) [2].

The main applications of VANET networks can be classified in three categories [3][4]:

\section{Application in prevention and road safety}

Road safety has become a priority in most developed countries. This priority becomes a necessity mainly with the increasing number of accidents related to the important number of vehicles used by citizens and road conditions. VANETs help to prevent collisions and work on the roads, to detect obstacles (fixed or mobile) and to distribute weather information by sending warning messages. VANETs can be used for example to alert a driver to the happening of an accident, and then he can exercise some prudence and forethought when heading to the accident either by changing his direction or doubling his vigilance. Alert and security messages must be brief to be transmitted as quickly as possible and must be issued at regular intervals.

\section{Application for traffic optimization and help in driving}

Car traffic can be greatly improved through the collection and sharing of data collected by the vehicles, which becomes a technical support for drivers. A car can, for example, be notified in case of abnormal slowdown situations (cork, traffic jam, rockslide or works) [5].

3.Applications for driver and passenger comfort

Vehicular networks can also improve the comfort of drivers and passengers. This comfort is illustrated by the internet access, messaging, inter-vehicle chat, etc. Passengers in the car can play in networks, download MP3 files, send cards to friends and access to other services [6]. 


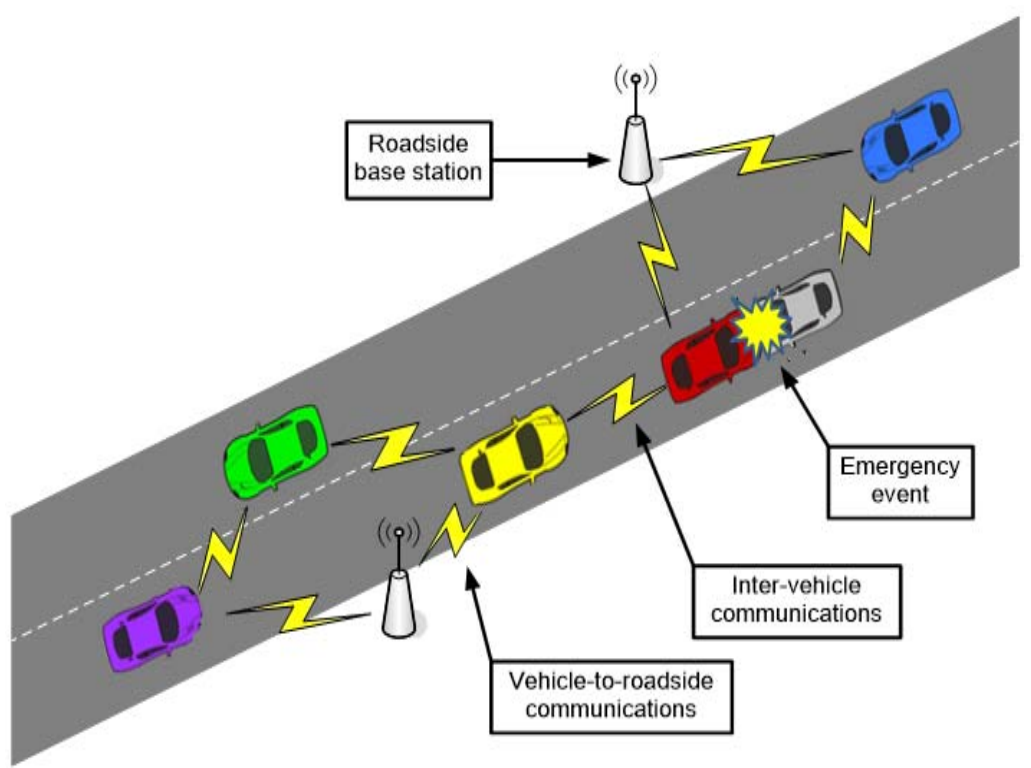

Fig 1. VANET Network

In this work, we are interested to present an approach based on a Multi-Agent Systems enabling a smart organization of a crossroads and road intersections, to make the road traffic management, in this complex places, more flexible and efficient. The Multi-agent Systems can avoid afterwards enormously problems, such as, accidents, loss of energy and time, as well as the traffic jam in the congestion situation.

This paper is organized as follows: Section 2 presents a brief definition of the Agent and Multi-agent systems. Section 3 introduces the SMA Framework used and proposed solution. Section 4 shows the effected simulation. Then we provide a conclusion in section 5.

\section{Agent And Multi-Agent SyStems}

An agent is an autonomous physical or abstract entity that is able to act and perceive about itself and its environment, which can communicate with other agents and whose behavior is the result of its observations, its knowledge, and interactions with other agents. The agent has its own resources and skills. The agent can both offer services and possibly reproduce them. [7][8].

A multi-agent system (MAS) is a community of autonomous agents evolved in a common environment (Fig.2), according to modes of cooperation, competition or even conflict, to achieve a global objective. These agents constitute a complex system that includes intelligence which could be described as collective. [9][10]

The agents in a multi-agent system have several important characteristics: [11]

- Autonomy: agents are partially independent, self-aware;

- Local views: no agent has a full global view;

- Decentralization: no agent is designated for controlling.

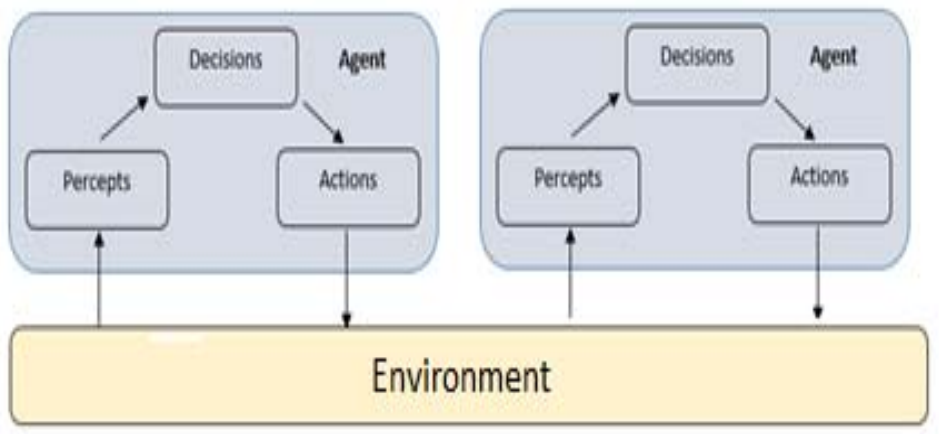

Fig.2 Multi-Agent System processes

Multi-agent systems can manifest self-organization, self-direction and other control paradigms. They can also relate complex behaviors even when the individual strategies of all their agents are simple.

MAS tend to find the best solution for their problems without any intervention. There is a high similarity here to physical phenomena, such as energy minimizing, where physical objects tend to reach the lowest energy 
possible within the physically constrained world [12]. The systems also tend to prevent propagation of faults, self-recover and be fault tolerant, mainly due to the redundancy of components [13].

MAS are applied in the real world to graphical applications such as computer games. Agent systems have already been used in films. They are used for coordinated defense systems. Other applications include transportation, [15] logistics, [14] graphics and GIS. It is widely advocated for use in networking and mobile technologies, to achieve automatic and dynamic load balancing, high scalability and self-healing networks.

MAS have also applications in the field of artificial intelligence where they reduce the complexity of solving a problem by dividing the necessary knowledge into subsets, by associating an independent intelligent agent with each of these subsets and coordinating the activity of these agents. This is called distributed artificial intelligence [16].

\section{SMA FRAMEWORK USED AND PROPOSED SOLUTION}

\section{A. SMA Framework used}

There are many platforms for the development of SMA, for example: TuCSoN, Pandora, Jadex, Ecolab, DESIRE JADE ... etc. These platforms differ from each other, according to several criteria such as, configuration, regularity of update, programming language used, distributed and parallel execution, GUI, compilation, scalability, communications between agents, complex calculations etc. [17].

We have opted for our work for the Java Agent Development framework (JADE) for the benefits it provides. [18].

JADE is a middleware that facilitates the development of multi-agent systems. It includes:

- A runtime environment where JADE agents can "live" and that must be active on a given host before one or more agents can be executed on that host;

- A library of classes whose programmers have to develop their agents;

- A suite of graphical tools that allows administrating and monitoring the activity of running agents.

\section{A.1. Containers and Platforms}

Each running instance of the JADE runtime environment is called a Container as it can contain several agents. The set of active containers is called a Platform. A single special Main container must always be active in a platform and all other containers registered with it as soon as they start. It follows that the first container to start in a platform must be a main container while all the other containers must be "normal" containers and must be informed where to find their main container to register with.

JADE agents are identified by a unique name and, provided they know each other's name, they can communicate transparently regardless of their actual location.

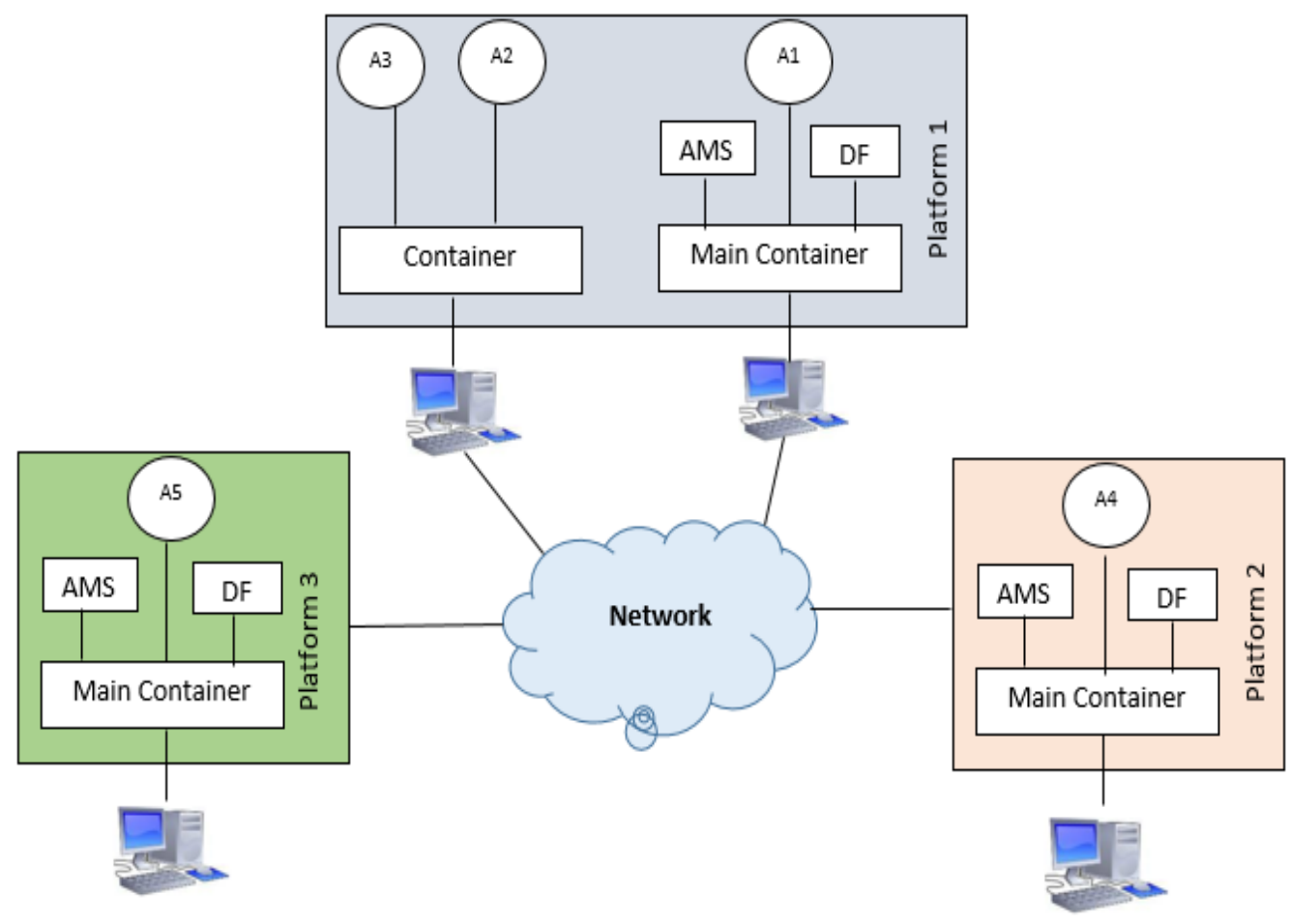

Fig. 3 Containers and Platforms 
- $\quad$ Same container (e.g. agents A2 and A3 in Fig.3);

- Different containers in the same platform (e.g. A1 and A2);

- Different platforms (e.g. A4 and A5).

A.2. AMS and DF

A main container differs from normal containers as it holds two special agents that automatically start functioning when the main container is launched.

Agent Management System (AMS) provides the naming service (to ensure for example that each agent has a unique identifier in the platform) and which represents the authority of the platform (for example it is possible to create / stop agents in sending requests to the AMS).

DF (Directory Facilitator) provides a yellow-page system that allows agents to find agent service providers.

\section{B. Proposed solution}

In the VANET environment, vehicles and traffic light, considered as mobile nodes, should be equipped with an On Board Unit. Therefore, we implement in each node an Agent (Fig.4).

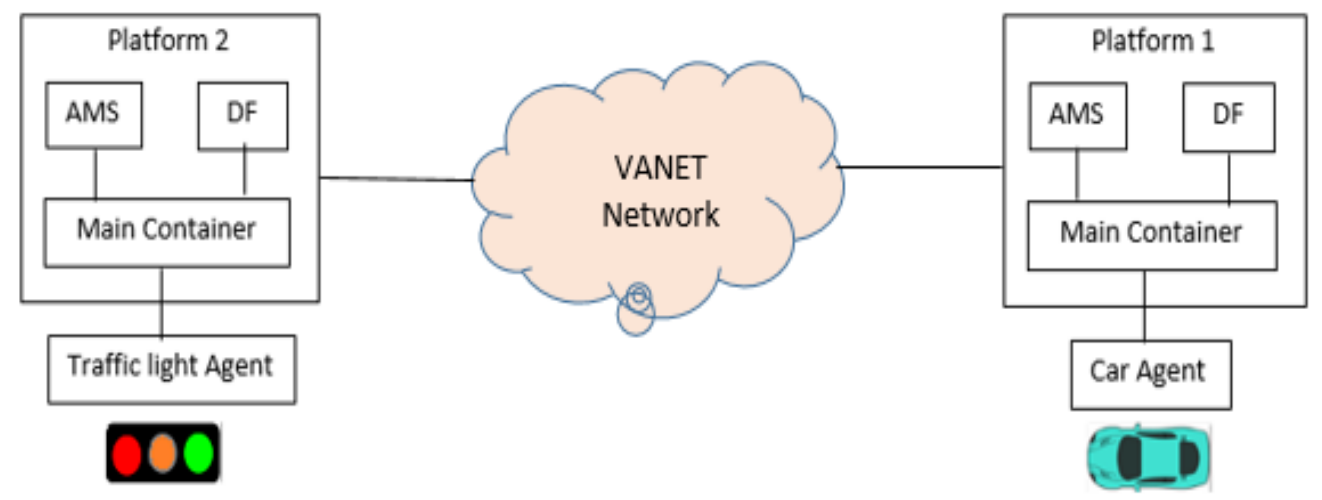

Fig. 4 Architecture of the proposed approach

Therefore, we create an intelligent distributed mobile network that can communicate, share information, analyze and make decisions.

In order to allow agents to collaborate, they must exchange messages. Each JADE agent has a sort of mailbox that contains messages sent to it by other agents. These mailboxes are in the form of a list that contains the messages in chronological order of their arrival.

JADE agents use messages that conform to FIPA ACL (FIPA Agent Communication Language) specifications [19].

In our case, managing a hub, we adopt two possible communications:

\section{1- Traffic light Agent - Car Agent Communication}

This communication can be realized in two different ways:

a- The traffic light broadcast its status to the different vehicles in front of its lane. The vehicles concerned are therefore just receivers and they will react according to the received state (slow down, stop or pass) (Fig.5).

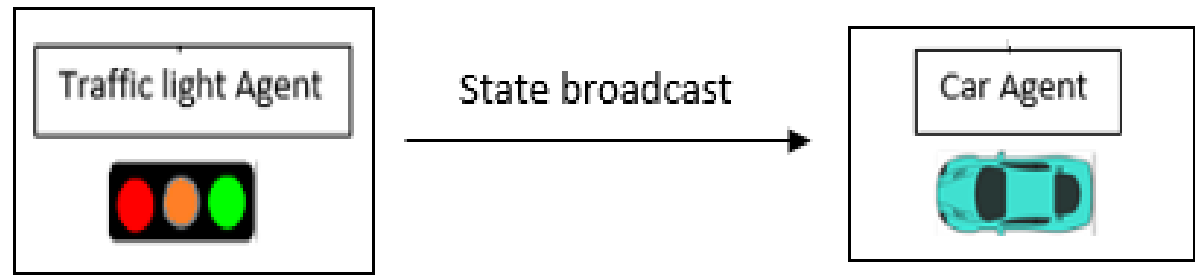

Fig.5 Broadcast agent communication

b- The vehicles send messages, in unicast, to the traffic light opposite to ask him for his condition. He replies thereafter, always in unicast, for each request. After receiving the traffic light status, the vehicles will make a suitable decision (slow down, stop or pass) (Fig.6). 




Fig.6 Unicast agent communication

\section{2- Traffic light Agent - Traffic light Agent Communication}

Following the internationally recognized road code, and especially in the management of intersections and crossroads, we have adopted a preprogrammed synchronous communication which consists in opposing the traffic light status according to the lanes (If the traffic light state of the horizontal lane is green, the others of the vertical lane will turn red) (Fig.7).

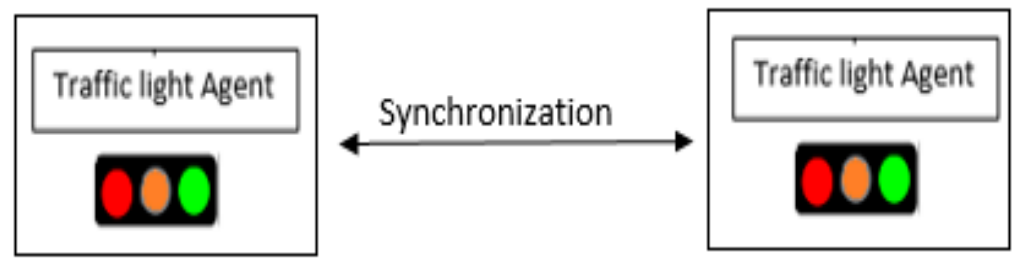

Fig.7 Traffic light Agents communication

\section{Algorithm of communication}

Traffic light is an active device used to send messages containing its status (Red, Green ...) in radio wave format. Suppose its transmission radius is $\mathrm{R}$, when a vehicle enters its coverage area $(\mathrm{d}<=\mathrm{R})$ the communication begins between these two agents according to the algorithm below (Fig.8).



Fig 8. Communication distances

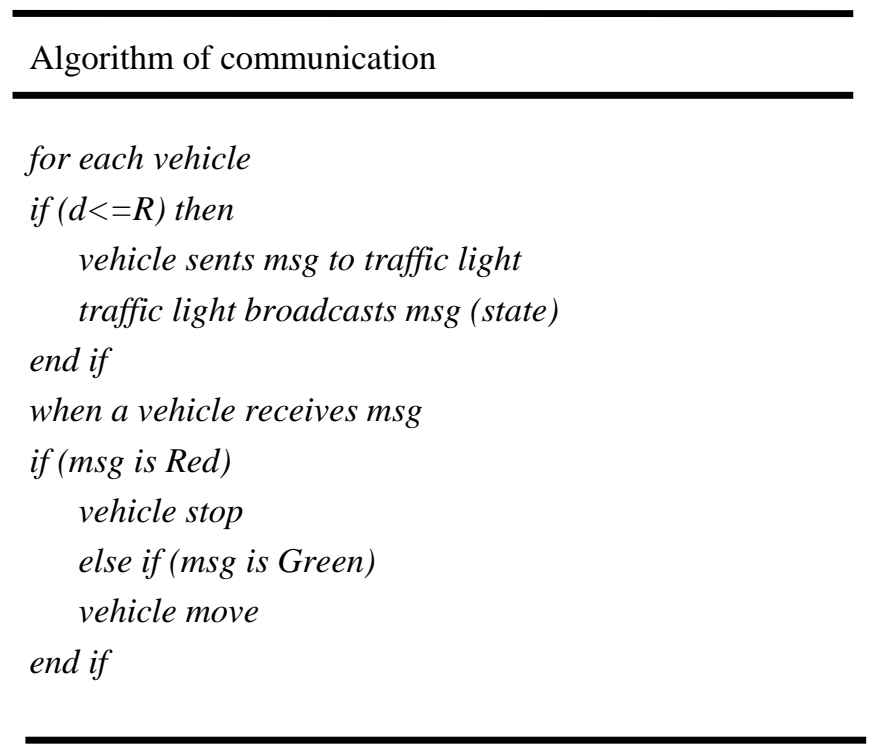




\section{Simulation}

To simulate our approach we have developed an application with java language using JADE Framework (Java Agent DEvelopment).

As mentioned above, we have created two types of agents: Car Agent and Traffic light Agent.

The Topology is a crossroads composed of two roads (two lanes in each road) and consists of four Traffic light and vehicles deployed in the different tracks.

Due to the communication between the various agents, the management of this complex place has become more flexible and smarter which makes it possible to avoid several problems like eventual accidents, waste of time and energy, pollution and the traffic jam in the congestion circumstances.

The figure below (Fig.9) shows the code programmed for the reaction of a vehicle agent when it receives a message broadcasted by the traffic light agent:



Fig.9 Receiving a message sent by a Traffic light

The different messages exchanged between the different agents (Traffic light and the vehicles) are shown in the figure below (Fig.8). This figure is the sniffer tool provided by the JADE framework:



Fig. 10 Exchanging messages between agents 
The execution of the simulation (Fig.11 and Fig.12) shows the validity of our approach, providing an intelligent and adequate mechanism. Due to communication between traffic lights agents and vehicles agent, crossroads management has become more flexible and more secure, thus avoiding several problems.

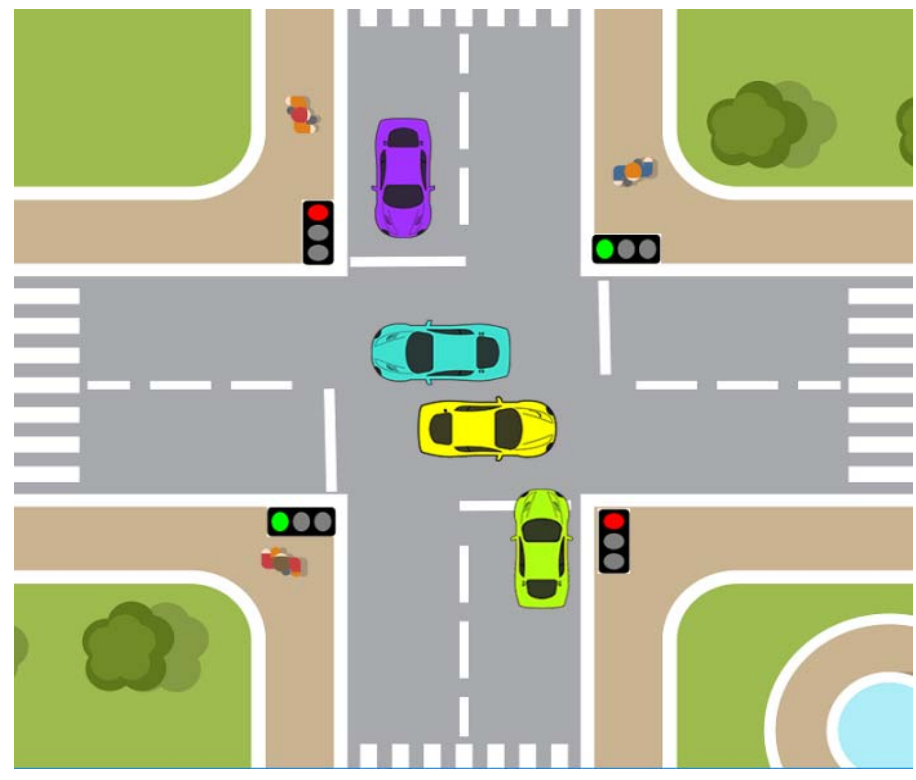

Fig.11 Cars passed when traffic light is green

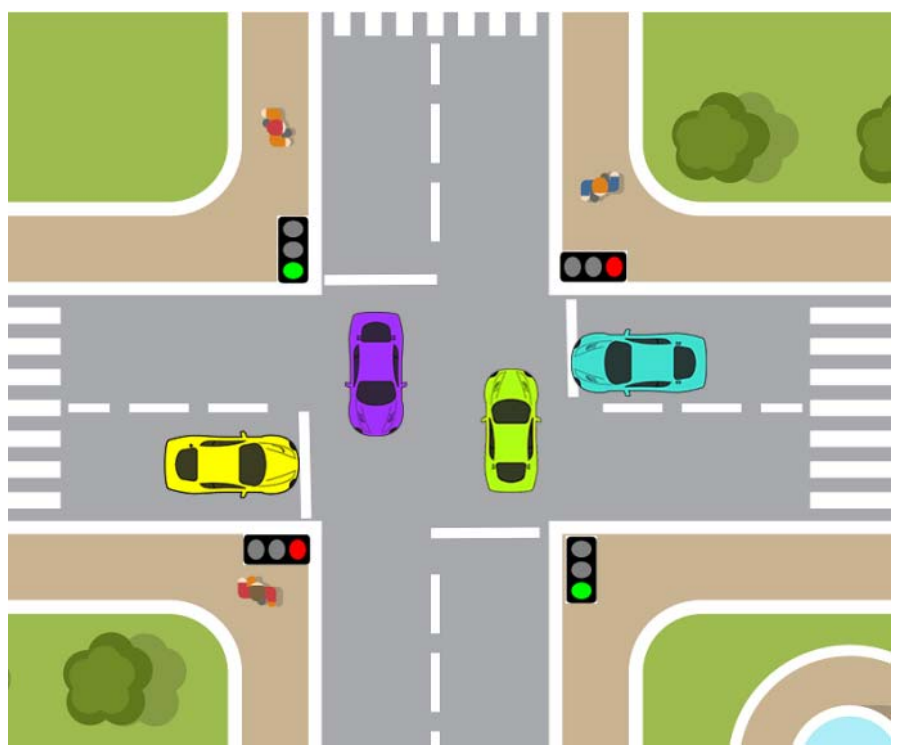

Fig.12 Cars stopped when traffic light is red

\section{CONCLUSION}

Although vehicle networks (VANET) are beginning to be widely spread out, the installation of various equipment, improvement of smart vehicles by adding numerous modules (GPS, sensors, computer, radar ...) and their operations have not reached a desired level. In this paper, we have proposed an intelligent approach based on Multi-agents technology permitting an intelligent management of intersections and crossroads. With this mechanism, the different nodes involved in the network will be able to communicate, exchange information and make the right decisions in a distributed and collaborative way. Moreover, the mechanism will produce more flexibility to the road traffic management and more efficiency in this complex places. This proposed approach will allow avoidance enormous problems, such as accidents, loss of energy and time as well as the traffic jam in the congestion state. The simulation test has shown that our approach has performed well by providing a harmonious environment of communication between different nodes.

Our future work will be to implement this approach in a real world to check its validity and its suitability and especially the reaction of the drivers' behavior that the simulators do not take them into consideration. 


\section{REFERENCES}

[1] H. Hartenstein and K. Laberteaux, Eds., VANET: Vehicular Applications and Inter-Networking Technologies. Wiley, 2010.

[2] K. C. Dey, A. Rayamajhi, M. Chowdhury, P. Bhavsar, and J. Martin, "Vehicle-to-vehicle (V2V) and vehicle-to-infrastructure (V2I) communication in a heterogeneous wireless network - Performance evaluation,” Transportation Research Part C: Emerging Technologies, vol. 68, pp. 168-184, 2016.

[3] Raju Barskar, Meenu Chawla, Vehicular Ad hoc Networks and its Applica-tions in Diversified Fields, International Journal of Computer Applications (0975 8887) Volume 123 No.10, August 2015.

[4] P.Pereira, A. Casaca, J.Rodrigues, V. Soares, J. Triay, C.Cervello-Pastor,” From Delay-Tolerant Networks to Vehicular DelayTolerant Networks,” Communications Surveys \& Tutorials, IEEE, Vol.14,Issue.4, pp. 1166 -1182,2012.

[5] S.Al-Sultan, M.M. Al-Doori ,A.H. Al-Bayatti , H. Zedan,” A comprehensive survey on vehicular Ad Hoc network,” Journal of Network and Computer Applications, Vol. 37, pp.380-392, 2014.

[6] H.Hartenstein, K.P.Laberteaux, "A tutorial survey on vehicular ad hoc networks," Communications Magazine, IEEE, Vol.46, Issue. 6, pp. 164 - 171, 2008.

[7] Julie A. Adams. Multiagent Systems: A modern approach to distributed artificial intelligence. AI Magazine, 22(2) :105-108, 2001.

[8] Ferber, J. (1999). Multi-agent systems: An introduction to distributed artificial intelligence. Harlow, UK: Addison-Wesley

[9] Chopra, N., \& Spong, M. W. (2006). Passivity-based control of multi-agent systems. In Advances in Robot Control (pp. 107-134). Berlin, Heidelberg: Springer.

[10] Nicholas R.Jennings “On agent-based software engineering” Artificial Intelligence, Volume 117, Issue 2, Pages 277-296, March 2000,

[11] Wooldridge, Michael (2002). An Introduction to MultiAgent Systems. John Wiley \& Sons. p. 366. ISBN 0-471-49691 X

[12] Orcun. Y, Geylani. K “A multi-agent system for minimizing energy costs in cement production” Computers in Industry, Volume 65, Issue 7, Pages 1076-1084, September 2014

[13] Oprea, M.: “Applications of multi-agent systems, in: Reis, R. (ed.): Information Technology, Kluwer Academic Publishers, Boston Dordrecht London (2004) 239-270.

[14] Xiao-Feng Xie, S. Smith, G. Barlow. "Schedule-driven coordination for real-time traffic network control”. International Conference on Automated Planning and Scheduling (ICAPS), São Paulo, Brazil, 2012: 323-331.

[15] Máhr, T. S., Srour, J., De Weerdt, M., Zuidwijk, R. (2010). "Can agents measure up? A comparative study of an agent-based and online optimization approach for a drayage problem with uncertainty". Transportation Research Part C: Emerging Technologies. 18: 99. doi:10.1016/j.trc.2009.04.018.

[16] Ponomarev, S., Voronkov, A. "Multi-agent systems and decentralized artificial superintelligence”. arXiv preprint arXiv:1702.08529 (2017)

[17] A. Rousset, B. Herrmann, C. Lang and L. Philippe "A survey on parallel and distributed multi-agent systems for high performance computing simulations” Computer Science Review, http://dx.doi.org/10.1016/j.cosrev.2016.08.001 1574-0137/@ 2016 Elsevier Inc.

[18] Bellifemine, F., Poggi, A., \& Rimassa, g. (2001). Developing multi-agent systems with a FIPA-compliant agent framework. SoftwarePractice and Experience, 31, 103-128.

[19] FIPA, Geneva, Switzerland, FIPA ACL Message Structure Specification, 2000/08/01 edition (August 2000) 